setbacks recently (21), the overwhelming clinical need for these interventions is clear. A definition of affordable, safe, and effective therapeutic intervention that can enhance $T$ cell renewal is clearly a challenge worth pursuing.

Address correspondence to: Steven G. Deeks, University of California, Ward 84, Building 80, 995 Potero Avenue, San Francisco, California 94110, USA. Phone: (415) 476-4082 ext. 404; Fax: (415) 476-6953; E-mail: sdeeks@php.ucsf.edu.

1. Sauce, D., et al. 2009. Evidence of premature immune aging in patients thymectomized during early childhood. J. Clin. Invest. 119:3070-3078.

2. Prelog, M., et al. 2009. Thymectomy in early childhood: significant alterations of the CD4(+) CD45RA(+)CD62L $(+) \mathrm{T}$ cell compartment in later life. Clin. Immunol. 130:123-132.

3. Hadrup, S.R., et al. 2006. Longitudinal studies of clonally expanded CD8 T cells reveal a repertoire shrinkage predicting mortality and an increased number of dysfunctional cytomegalovirusspecific $T$ cells in the very elderly. J. Immunol. 176:2645-2653.

4. Wikby, A., et al. 2006. The immune risk phenotype is associated with IL-6 in the terminal decline stage: findings from the Swedish NONA immune longitudinal study of very late life functioning. Mech. Ageing Dev. 127:695-704.

5. Strindhall, J., et al. 2007. No Immune Risk Profile among individuals who reach 100 years of age: findings from the Swedish NONA immune longitudinal study. Exp. Gerontol. 42:753-761.

6. Deayton, J.R., et al. 2004. Importance of cytomegalovirus viraemia in risk of disease progression and death in HIV-infected patients receiving highly active antiretroviral therapy. Lancet. 363:2116-2121.

7. Hsue, P.Y., et al. 2006. Increased carotid intimamedia thickness in HIV patients is associated with increased cytomegalovirus-specific T-cell responses. AIDS. 20:2275-2283.

8. Peggs, K.S., et al. 2003. Reconstitution of T-cell repertoire after autologous stem cell transplantation: influence of CD34 selection and cytomegalovirus infection. Biol. Blood Marrow Transplant. 9:198-205.

9. Sylwester, A.W., et al. 2005. Broadly targeted human cytomegalovirus-specific CD4+ and CD8+ T cells dominate the memory compartments of exposed subjects. J. Exp. Med. 202:673-685.

10. Bourgeois, C., Hao, Z., Rajewsky, K., Potocnik, A.J., and Stockinger, B. 2008. Ablation of thymic export causes accelerated decay of naive CD4 T cells in the periphery because of activation by environmental antigen. Proc. Natl. Acad. Sci.U. S. A. 105:8691-8696.

11. Napolitano, L.A., et al. 2008. Growth hormone enhances thymic function in HIV-1-infected adults. J. Clin. Invest. 118:1085-1098.

12. Barton, E.S., et al. 2007. Herpesvirus latency confers symbiotic protection from bacterial infection. Nature. 447:326-329.

13. Nikolich-Zugich, J. 2008. Ageing and life-long maintenance of T-cell subsets in the face of latent persistent infections. Nat. Rev. Immunol. 8:512-522.

14. Pawelec, G., et al. 2004. Is immunosenescence infectious? Trends Immunol. 25:406-410.

15. Hakim, F.T., et al. 2005. Age-dependent incidence, time course, and consequences of thymic renewal in adults. J. Clin. Invest. 115:930-939.

16. Chu, Y.W., et al. 2008. Exogenous insulin-like growth factor 1 enhances thymopoiesis predominantly through thymic epithelial cell expansion. Blood. 112:2836-2846.

17. Williams, K.M., et al. 2008. CCL25 increases thymopoiesis after androgen withdrawal. Blood. 112:3255-3263

18. Sutherland, J.S., et al. 2005. Activation of thymic regeneration in mice and humans following androgen blockade. J. Immunol. 175:2741-2753.

19. Sportes, C., et al. 2008. Administration of rhIL-7 in humans increases in vivo TCR repertoire diversity by preferential expansion of naive $\mathrm{T}$ cell subsets. J. Exp. Med. 205:1701-1714.

20. Levy, Y., et al. 2009. Enhanced T cell recovery in HIV-1-infected adults through IL-7 treatment. J. Clin. Invest. 119:997-1007.

21. Kuritzkes, D.R. 2009. Interleukin-2: trials and tribulations. J. Infect. Dis. 200:164-165.

\title{
Pin1 regulates parathyroid hormone mRNA stability
}

\author{
Rajiv Kumar \\ Division of Nephrology and Hypertension, Departments of Medicine, Biochemistry, and Molecular Biology, \\ Mayo Clinic and Foundation, Rochester, Minnesota, USA.
}

\begin{abstract}
Secondary hyperparathyroidism often occurs in chronic kidney disease (CKD) and vitamin $\mathrm{D}$ deficiency, resulting in increased fractures and mortality. Understanding factors that stimulate parathyroid hormone (PTH) synthesis is important for devising methods to treat this condition. Previous work has demonstrated that murine $P$ th $\mathrm{mRNA}$ levels are regulated by proteins that bind AU-rich elements (AREs) within the $3^{\prime}$ UTR region of $P t h$ mRNA and influence Pth mRNA stability. In this issue of the JCI, Nechama et al. demonstrate that in murine secondary hyperparathyroidism associated with CKD or Ca deficiency, the activity of Pin1, a peptidyl-prolyl isomerase, is reduced (see the related article beginning on page 3102). Reduced Pin 1 activity resulted in the phosphorylation and degradation of an ARE-binding protein, K-homology splicing regulator protein (KSRP), which normally enhances the degradation of $P t h$ mRNA. The activity of other ARE-binding proteins, such as AU-rich binding factor 1 (AUF1), that increase Pth mRNA stability, was increased, thereby increasing PTH synthesis. This work suggests new ways by which to regulate PTH synthesis in secondary hyperparathyroidism.
\end{abstract}

Conflict of interest: The author has declared that no conflict of interest exists.

Citation for this article: J. Clin. Invest. 119:2887-2891 (2009). doi:10.1172/JCI40784.

\section{RNA processing in the cytoplasm regulates RNA concentrations}

Following transcription, nascent RNA is processed by $5^{\prime}$ methyl capping, splicing, cleavage, and polyadenylation in the nucleus (1-4) (Figure 1). RNA is exported from the nucleus and associates with various cellular structures prior to association with the ribosome. In the cytoplasm, RNA transcripts interact with RNA-binding proteins that influence RNA half-life and stability within the cell (5-8) (Figure 1). RNA-binding proteins (Table 1) associate with sequence-specific elements (adenine- and uridine-rich elements [AREs]) either within the coding or, more usually, within the $3^{\prime}$ UTRs of RNA. AREs regulate the rate at which mRNAs are degraded in cells and were first described as important elements involved in the regulation of the stability and half-life of protooncogene and cytokine mRNAs (1,9-12). AREs often contain overlapping adenine- and uridine-containing AUUUA pentamers that are found in U-rich regions within the $3^{\prime}$ UTRs of various genes (13). Three classes of AREs have been described: class I AREs contain 


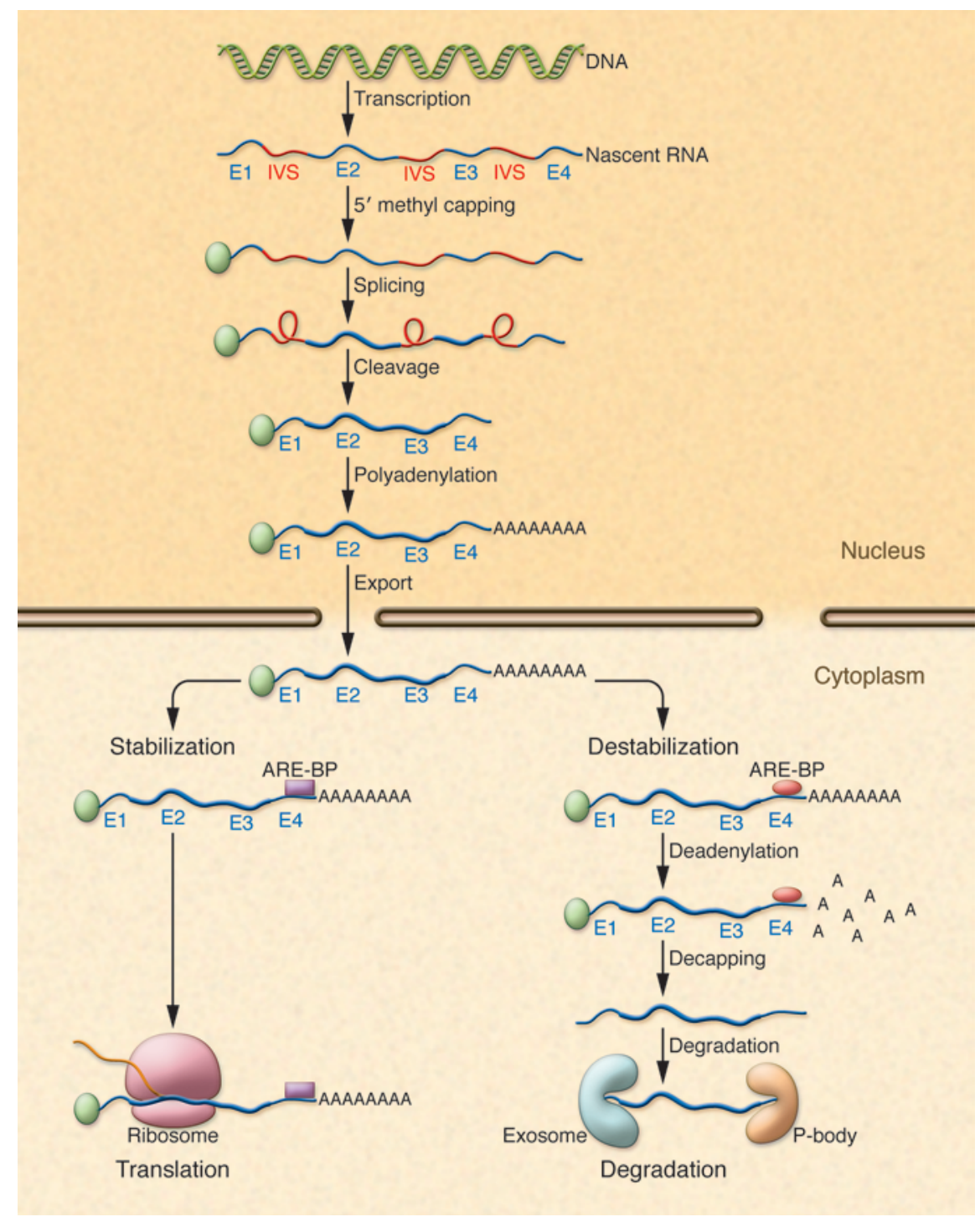

several copies of the AUUUA motif dispersed within U-rich regions; class II AREs possess at least two overlapping UUAUUUA(U/A) nonamers; and class III AREs are less well defined and generally do not contain an AUUUA sequence $(1,10,13)$. Whether an mRNA species containing an ARE bound to ARE-binding proteins (ARE-BPs) is degraded or stabilized is partly dependent upon the cellular milieu, physiological circumstances, and the relative amounts of different bound stabilizing or destabilizing ARE-BPs. Following binding of ARE-BPs to an ARE, RNAs are targeted for translation or degradation. RNAs targeted for degradation undergo deadenylation, decapping, and degradation in a large multiprotein complex, the exosome, or in cytoplasmic compartments known as GW bodies or processing bodies (P-bodies) (14-16).

\section{Parathyroid hormone serum concentrations are dependent on parathyroid hormone secretion and synthesis}

The parathyroid (PT) glands play a central role in $\mathrm{Ca}$ homeostasis by regulating bone resorption and formation, the synthesis of $1 \alpha, 25$-dihydroxyvitamin $\mathrm{D}$ in the renal proximal tubule, and the reabsorption of $\mathrm{Ca}^{2+}$ in the distal nephron of the kidney (17-21). Changes in serum $\mathrm{Ca}^{2+}$ concentrations are sensed by PT chief cells via a cell-surface, G protein-coupled receptor, the $\mathrm{Ca}^{2+}$-sensing receptor, and result in rapid (within minutes) alterations in parathyroid hormone (PTH) secretion $(22,23)$. More long-term changes in serum $\mathrm{Ca}^{2+}$ concentrations (over several hours) result in increases or decreases in PTH synthesis and PTH mRNA concentrations in the PT gland $(24,25)$.

\section{Figure 1}

Cellular processing of RNA. Following transcription, nascent RNA comprised of exons (E1-E4) and intervening sequences (IVS) is processed in the nucleus by $5^{\prime}$ methyl capping, splicing, cleavage, and polyadenylation. Processed RNA is exported from the nucleus and binds various structural elements and binding proteins. ARE-BPs (purple box and red oval) bind to AREs within the $3^{\prime}$ region of RNA and stabilize or destabilize mRNA. Stabilized RNA undergoes translation in ribosomes, whereas destabilized RNA undergoes deadenylation, decapping, and degradation in exosomes or P-bodies.

\section{Pathogenesis of secondary hyperparathyroidism}

Secondary hyperparathyroidism occurs in the clinical context of vitamin $\mathrm{D}$ deficiency, Ca deficiency, and chronic kidney disease (CKD) $(26,27)$. The pathogenesis of secondary hyperparathyroidism in CKD is multifactorial and includes phosphate retention and hyperphosphatemia, hypocalcemia, $1 \alpha, 25$-dihydroxyvitamin D deficiency, intestinal Ca malabsorption, the reduction in vitamin $\mathrm{D}$ receptor concentrations within the PT gland, and reduced $\mathrm{Ca}^{2+}$-sensing receptor amounts in PT tissue (28-30). Not only is PTH synthesis increased with concomitant increases in serum PTH concentrations, but PT hyperplasia often occurs as well $(29,30)$. In CKD and dialysis patients, uncontrolled secondary hyperparathyroidism is associated with an increased incidence of fractures and increased mortality (31-34). Numerous methods, including the control of serum phosphate concentrations, the administration of vitamin $\mathrm{D}$ analogs, Ca supplementation, and the administration of calcimimetics, have been developed to control PTH levels in CKD and dialysis patients (35-38), but secondary hyperparathyroidism in CKD remains a significant problem. Additional methods for the treatment of this condition would therefore be of value.

\section{Pth mRNA amounts are regulated by $\mathrm{Ca}^{2+}$ and $\mathrm{Pi}$ by posttranscriptional mechanisms}

In murine PT glands, changes in Pth mRNA concentrations following alterations in serum $\mathrm{Ca}^{2+}$ (or inorganic phosphate, $\mathrm{Pi}$ ) concentrations are due to alterations in $P$ th mRNA stability rather than changes in Pth mRNA transcription (39, 40). Such changes are brought about by the binding of proteins to the terminal 


\section{Table 1}

Effect of ARE-BPs on mRNA stability

\section{ARE-BP}

AUF1 (HNRNPD, heterogenous nuclear ribonucleoprotein D0)

HuR (Hu-antigen R, ELAV-like protein 1)

Hel-N1 (Hu-antigen B, ELAV-like protein 2)

HuD (Hu-antigen D, ELAV-like protein 4)

KSRP (far upstream element-binding protein 2, FUSE-binding protein 2)

TTP (tristetraproline)

BRF-1 (B-related factor 1, transcription factor IIIB $90 \mathrm{kDa}$ subunit)

CUG-BP2 (CUG triplet repeat RNA-binding protein 2, CUG-BPand ETR-3-like factor 2, Bruno-like protein 3, RNA-binding protein BRUNOL-3, ELAV-type RNA-binding protein 3, neuroblastoma apoptosis-related RNA-binding protein)

Nucleolin

TINO (RNA-binding protein MEX3D, RING finger- and

KH domain-containing protein 1, RING finger protein 193)

PAIP2 (Polyadenylate-binding protein-interacting protein 2)

\section{Effect on stability of mRNA of various genes \\ Increase}

c-myc, c-fos, PTH, GMCSF, TNFA

c-myc, c-fos, MyoD, cyclin A, cyclin B1, cyclin D1, NOS2, GMCSF, TNFA, COX2,

$$
\begin{gathered}
\text { IL3, VEGF, myogenin } \\
\text { TNFA, GLUT1 } \\
\text { GAP43 }
\end{gathered}
$$

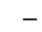

c-fos, NOS2, TNFA, IL2, c-Jun, PTH

$-$

c-fos, GMCSF, TNFA, COX2, IL3, IL2

$-$

$\operatorname{cox} 2$

$\mathrm{Bcl}-2$

- $\quad B c /-2$

VEGF
TNFA, IL3

c-myc, c-fos, p21, cyclin D1, GMCSF, IL3
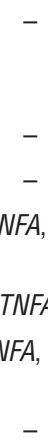

Modified with permission from Nucleic Acids Research (9).

portion of the 3' UTR of Pth mRNA (Figure 2). A 63-nt ARE in the $3^{\prime}$ UTR regulates Pth mRNA stability in response to changes in $\mathrm{Ca}^{2+}$ and Pi concentrations $(39,41,42)$. The 63-nt element consists of a core 26-nt minimal binding sequence and adjacent flanking regions. The Pth RNA ARE does not contain AUUUA sequences and falls into the class III category of AREs $(42,43)$. Two proteins, AU-rich binding factor 1 (AUF1) and K-homology splicing regulator protein (KSRP), bind the ARE in the $3^{\prime}$
UTR of $P$ th mRNA $(43,44)$. AUF1 increases $P$ th mRNA half-life, whereas KSRP has the opposite effect $(43,44)$. Both proteins are regulated by changes in serum $\mathrm{Ca}^{2+}$ and $\mathrm{Pi}$ concentrations and are altered by $\operatorname{CKD}(43,45)$.

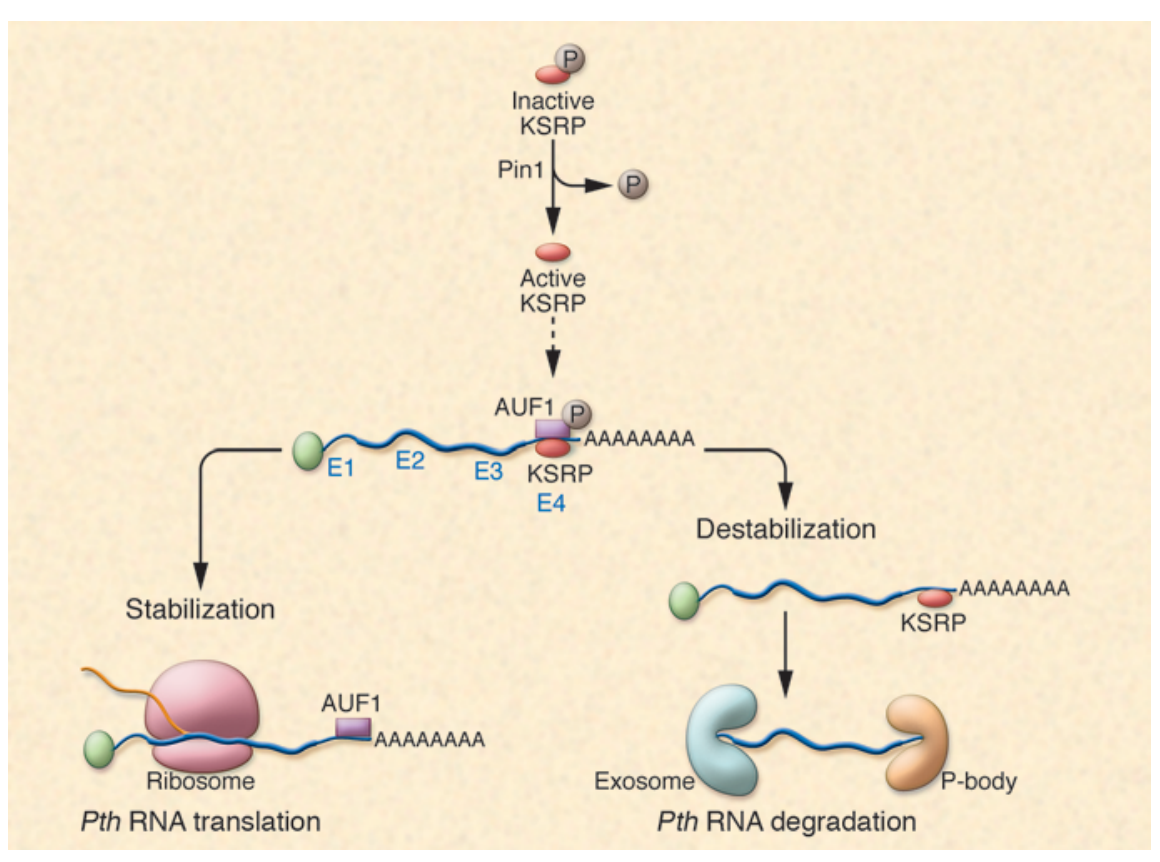

\section{Figure 2}

Processing of Pth mRNA. Murine Pth mRNA is bound by ARE-BPs, which either stabilize or destabilize Pth mRNA, thereby altering $P$ th mRNA half-life. The ratio of activities of stabilizing/destabilizing ARE-BPs bound to Pth mRNA determines the half-life of a given $P$ th mRNA molecule. KSRP is a $P$ th mRNA-destabilizing ARE-BP that is active in its dephosphorylated state. In their new study in this issue of the $\mathrm{JCl}$, Nechama et al. (46) report that the peptidyl-prolyl isomerase Pin1 is responsible for the dephosphorylation of KSRP. In CKD, Pin1 activity is reduced, and as a result, less dephosphorylated (active) KSRP is available. As a consequence, a stabilizing ARE-BP, AUF1, is active and Pth mRNA is degraded to a lesser extent, resulting in higher intracellular Pth mRNA levels, more PTH synthesis, and secondary hyperparathyroidism. $\mathrm{P}$, phosphate. 


\section{Pin1, a peptidyl-prolyl isomerase, alters KSRP phosphorylation and binding of KSRP to the Pth ARE}

In the accompanying article, Nechama et al. have elegantly dissected the manner in which Pth mRNA is degraded via AREs and cognate ARE-BPs in secondary hyperparathyroidism in rodents and have shown a role for the enzyme peptidyl-prolyl cis/trans isomerase NIMA-interacting 1 (Pin 1 ) in this process (46) (Figure 2). The authors demonstrate that the secondary hyperparathyroidism associated with CKD or Ca deficiency is due in part to reduced Pin 1 activity in the PT glands. The reduction in Pin 1 activity reduced the ratio of the ARE-BPs, KSRP, and AUF1, which normally exert opposite effects on $P$ th mRNA stability. As a result, Pth mRNA half-life and stability were increased due to unopposed AUF1 activity. The data suggest that it is possible to modulate Pth mRNA halflife and stability by altering the activity of Pin 1 and by changing KSRP concentrations within the PT cell.

Pin1 is a peptidyl-prolyl isomerase that specifically binds serine/threonine-protein motifs and catalyzes the cis-trans isomerization of peptide bonds, thereby changing the activity of proteins $(47,48)$. Previous work from other laboratories has shown that Pin1 interacts with AUF1 and stabilizes GMCSF and TGFB mRNAs (49, 50). Nechama et al. (46) hypothesized that Pin 1 might also alter Pth mRNA stability and play a role in the pathogenesis of secondary hyperparathyroidism seen in CKD. They showed the presence of Pin 1 epitopes and Pin 1 enzymatic activity in PT glands and PT extracts. Induction of secondary hyperparathyroidism by feeding rats a diet low in Ca or by inducing CKD with adenine reduced Pin 1 activity. Reduced Pin 1 activity correlated with increased $P$ th mRNA levels in the PT glands of such animals. Inhibition of Pin 1 activity with the inhibitor juglone increased $P t h$ mRNA levels. The increase in Pth mRNA levels in juglonetreated PT was posttranscriptional, since nuclear run-on experiments revealed no changes in the transcription rate of Pth. In a surrogate cell line, HEK293 (a PT cell line is not available for transfection experiments), Pin 1 overexpression accelerated $P t h$ mRNA decay, whereas Pin 1 knockdown with siRNA decreased Pth mRNA decay. Also, Pin 1 overexpression was without effect on the half-life of a Pth transcript lacking the ARE-containing Pth 3' UTR. Both the protein-interaction domain and the peptidyl-prolyl cis-trans isomerization domains of Pin 1 were necessary for the effect of this protein on Pth mRNA stability. Interestingly, when the 63-nt Pth ARE was introduced into the $3^{\prime}$ UTR of a growth hormone reporter gene (GH63nt), Pin 1 overexpression decreased chimeric GH mRNA levels. Treatment with juglone of cells transfected with such a construct decreased Pin 1 activity and increased GH63-nt mRNA levels. Pin 1 bound KSRP, an ARE-BP that increases the degradation of $P t h$ mRNA. In cells transfected with a chimeric GH63-nt reporter containing the Pth 63-nt ARE, KSRP overexpression decreased GH63-nt mRNA levels. Conversely, KSRP depletion increased GH63-nt mRNA levels in the presence of Pin 1 expression. In such cells, Pin 1 inhibition prevented KSRPmediated decreases in GH63-nt mRNA. The authors also demonstrated that inhibition of Pin 1 by juglone in PT glands in vivo reduced KSRP binding to Pth mRNA, thus increasing Pth mRNA half-life. Nechama et al. showed that Pin 1 prevents the phosphorylation of KSRP at serine residue 181 and that a mutant KSRP (S181A) that is incapable of being phosphorylated had increased activity. Pin1-knockout mice had increased PT gland PTH levels and circulating serum PTH concentrations without changes in serum $\mathrm{Ca}$ and Pi levels.

In summary, the data reported in this issue by Nechama et al. (46) are consistent with a biological role for Pin 1 in the pathogenesis of secondary hyperparathyroidism in rat PT via regulation of the amount of active, nonphosphorylated KSRP in PT cells. Several additional areas of investigation remain to be explored. For example, it is unclear what triggers the reduction in Pin 1 activity in the parathyroids in chronic renal failure and $\mathrm{Ca}$ deficiency. Future studies might be directed at identifying factors that regulate Pin 1 activity and expression in the PT gland. Precise quantification of KSRP/AUF1 ratios in the PT gland in different conditions would also be of value. The development of PT gland-specific modulators of ARE-BPs might result in drugs that are effective for the control of secondary hyperparathyroidism and PT hyperplasia. Stay tuned for developments in this area.

\section{Acknowledgments}

The author's laboratory is supported by NIH grants DK76829 and DK77669 and a grant from Genzyme (Genzyme Renal Innovations Program [GRIP]).
Address correspondence to: Rajiv Kumar, Division of Nephrology and Hypertension, Departments of Medicine, Biochemistry and Molecular Biology, Mayo Clinic and Foundation, 200 1st St. SW, Rochester, Minnesota 55905, USA. Phone: 507284-0020; Fax: 507-538-9536; E-mail: rkumar@mayo.edu.

1. McKee, A.E., and Silver, P.A. 2007. Systems perspectives on mRNA processing. Cell Res. 17:581-590.

2. Bentley, D.L. 2005. Rules of engagement: co-transcriptional recruitment of pre-mRNA processing factors. Curr. Opin. Cell Biol. 17:251-256.

3. Blencowe, B.J. 2006. Alternative splicing: new insights from global analyses. Cell. 126:37-47.

4. Kornblihtt, A.R., de la Mata, M., Fededa, J.P., Munoz, M.J., and Nogues, G. 2004. Multiple links between transcription and splicing. RNA. 10:1489-1498.

5. Hieronymus, H., and Silver, P.A. 2004. A systems view of mRNP biology. Genes Dev. 18:2845-2860.

6. Moore, M.J. 2005. From birth to death: the complex lives of eukaryotic mRNAs. Science. 309:1514-1518.

7. Sanchez-Diaz, P., and Penalva, L.O. 2006. Posttranscription meets post-genomic: the saga of RNA binding proteins in a new era. RNA Biol. 3:101-109.

8. Singh, R., and Valcarcel, J. 2005. Building specificity with nonspecific RNA-binding proteins. Nat. Struct. Mol. Biol. 12:645-653.

9. Barreau, C., Paillard, L., and Osborne, H.B. 2005. AUrich elements and associated factors: are there unifying principles? Nucleic Acids Res. 33:7138-7150.

10. Misquitta, C.M., Chen, T., and Grover, A.K. 2006. Control of protein expression through mRNA stability in calcium signalling. Cell Calcium. 40:329-346.

11. Schiavi, S.C., Belasco, J.G., and Greenberg, M.E. 1992. Regulation of proto-oncogene mRNA stability. Biochim. Biophys. Acta. 1114:95-106.

12. Schiavi, S.C., et al. 1994. Multiple elements in the c-fos protein-coding region facilitate mRNA deadenylation and decay by a mechanism coupled to translation. J. Biol. Chem. 269:3441-3448.

13. Chen, C.Y., and Shyu, A.B. 1995. AU-rich elements: characterization and importance in mRNA degradation. Trends Biochem. Sci. 20:465-470.

14. Lebreton, A., Tomecki, R., Dziembowski, A., and Seraphin, B. 2008. Endonucleolytic RNA cleavage by a eukaryotic exosome. Nature. 456:993-996.

15. McPheeters, D.S., et al. 2009. A complex gene regulatory mechanism that operates at the nexus of multiple RNA processing decisions. Nat. Struct. Mol. Biol. 16:255-264.

16. Schilders, G., and Pruijn, G.J. 2008. Biochemical studies of the mammalian exosome with intact cells. Methods Enzymol. 448:211-226.

17. Habener, J.F., Rosenblatt, M., and Potts, J.T., Jr. 1984. Parathyroid hormone: biochemical aspects of biosynthesis, secretion, action, and metabolism. Physiol. Rev. 64:985-1053.

18. Potts, J.T. 2005. Parathyroid hormone: past and present. J. Endocrinol. 187:311-325.

19. Kumar, R. 1984. Metabolism of 1,25-dihydroxyvitamin D3. Physiol. Rev. 64:478-504.

20. DeLuca, H.F., and Schnoes, H.K. 1983. Vitamin D: recent advances. Annu. Rev. Biochem. 52:411-439.

21. Gesek, F.A., and Friedman, P.A. 1992. On the mechanism of parathyroid hormone stimulation of calcium uptake by mouse distal convoluted tubule cells. J. Clin. Invest. 90:749-758.

22. Hofer, A.M., and Brown, E.M. 2003. Extracellular calcium sensing and signalling. Nat. Rev. Mol. Cell Biol. 4:530-538.

23. Fox, J., and Heath, H., 3rd. 1981. The "calcium clamp": effect of constant hypocalcemia on parathyroid hormone secretion. Am. J. Physiol. 240:E649-E655.

24. Naveh-Many, T., and Silver, J. 1990. Regulation of 
parathyroid hormone gene expression by hypocalcemia, hypercalcemia, and vitamin D in the rat. J. Clin. Invest. 86:1313-1319.

25. Naveh-Many, T., Friedlaender, M.M., Mayer, H., and Silver, J. 1989. Calcium regulates parathyroid hormone messenger ribonucleic acid (mRNA), but not calcitonin mRNA in vivo in the rat. Dominant role of 1,25-dihydroxyvitamin D. Endocrinology. 125:275-280.

26. Audran, M., Gross, M., and Kumar, R. 1986. The physiology of the vitamin D endocrine system. Semin. Nephrol. 6:4-20.

27. Audran, M., and Kumar, R. 1985. The physiology and pathophysiology of vitamin D. Mayo Clin. Proc. 60:851-866.

28. Cozzolino, M., et al. 2005. Pathogenesis of parathyroid hyperplasia in renal failure. J. Nephrol. 18:5-8.

29. Dusso, A.S., et al. 2007. Molecular basis of parathyroid hyperplasia. J. Ren. Nutr. 17:45-47.

30. Dusso, A.S., et al. 2006. Pathogenic mechanisms for parathyroid hyperplasia. Kidney Int. Suppl. 102:S8-S11.

31. Danese, M.D., et al. 2006. PTH and the risks for hip, vertebral, and pelvic fractures among patients on dialysis. Am. J. Kidney Dis. 47:149-156.

32. Jadoul, M., et al. 2006. Incidence and risk factors for hip or other bone fractures among hemodialysis patients in the Dialysis Outcomes and Practice Patterns Study. Kidney Int. 70:1358-1366.

33. Rudser, K.D., de Boer, I.H., Dooley, A., Young, B., and Kestenbaum, B. 2007. Fracture risk after parathyroidectomy among chronic hemodialysis patients. J. Am. Soc. Nephrol. 18:2401-2407.

34. Block, G.A., et al. 2004. Mineral metabolism, mortality, and morbidity in maintenance hemodialysis.
J. Am. Soc. Nephrol. 15:2208-2218.

35. Block, G.A., et al. 2004. Cinacalcet for secondary hyperparathyroidism in patients receiving hemodialysis. N. Engl. J. Med. 350:1516-1525.

36. Drueke, T.B. 2004. Calcimimetics versus vitamin D: what are their relative roles? Blood Purif. 22:38-43.

37. Locatelli, F., et al. 2002. Management of disturbances of calcium and phosphate metabolism in chronic renal insufficiency, with emphasis on the control of hyperphosphataemia. Nephrol. Dial. Transplant. 17:723-731.

38. Moe, S.M., and Drueke, T.B. 2003. Management of secondary hyperparathyroidism: the importance and the challenge of controlling parathyroid hormone levels without elevating calcium, phosphorus, and calcium-phosphorus product. Am. J. Nephrol. 23:369-379.

39. Moallem, E., Kilav, R., Silver, J., and Naveh-Many, T. 1998. RNA-Protein binding and post-transcriptional regulation of parathyroid hormone gene expression by calcium and phosphate. J. Biol. Chem. 273:5253-5259.

40. Levi, R, et al. 2006. Increased parathyroid hormone gene expression in secondary hyperparathyroidism of experimental uremia is reversed by calcimimetics: correlation with posttranslational modification of the trans acting factor AUF1. J. Am. Soc. Nephrol. 17:107-112.

41. Naveh-Many, T., Sela-Brown, A., and Silver, J. 1999. Protein-RNA interactions in the regulation of PTH gene expression by calcium and phosphate. Nephrol. Dial. Transplant. 14:811-813.

42. Kilav, R., Bell, O., Le, S.Y., Silver, J., and Naveh-Many, T. 2004. The parathyroid hormone mRNA 3'-untranslated region AU-rich element is an unstructured func- tional element. J. Biol. Chem. 279:2109-2116.

43. Naveh-Many, T., Bell, O., Silver, J., and Kilav, R. 2002. Cis and trans acting factors in the regulation of parathyroid hormone (PTH) mRNA stability by calcium and phosphate. FEBS Lett. 529:60-64.

44. Nechama, M., Ben-Dov, I.Z., Briata, P., Gherzi, R., and Naveh-Many, T. 2008. The mRNA decay promoting factor K-homology splicing regulator protein post-transcriptionally determines parathyroid hormone mRNA levels. FASEB J. 22:3458-3468.

45. Nechama, M., Ben-Dov, I.Z., Silver, J., and NavehMany, T. 2009. Regulation of PTH mRNA stability by the calcimimetic R568 and the phosphorus binder lanthanum carbonate in CKD. Am. J. Physiol. Renal Physiol. 296:F795-F800.

46. Nechama, M., Uchida, T., Mor Yosef-Levi, I., Silver, J., and Naveh-Many, T. 2009. The peptidyl-prolyl isomerase Pin1 determines parathyroid hormone mRNA levels and stability in rat models of secondary hyperparathyroidism. J. Clin. Invest. 119:3102-3114.

47. Lu, K.P., Liou, Y.C., and Zhou, X.Z. 2002. Pinning down proline-directed phosphorylation signaling. Trends Cell Biol. 12:164-172.

48. Lu, K.P., and Zhou, X.Z. 2007. The prolyl isomerase PIN1: a pivotal new twist in phosphorylation signalling and disease. Nat. Rev. Mol. Cell Biol. 8:904-916.

49. Shen, Z.J., Esnault, S., and Malter, J.S. 2005. The peptidyl-prolyl isomerase Pin1 regulates the stability of granulocyte-macrophage colony-stimulating factor mRNA in activated eosinophils. Nat. Immunol. 6:1280-1287.

50. Shen, Z.J., et al. 2008. Pin 1 regulates TGF-beta1 production by activated human and murine eosinophils and contributes to allergic lung fibrosis. J. Clin. Invest. 118:479-490.

\title{
Resolving lung injury: a new role for Tregs in controlling the innate immune response
}

\author{
Anthony Pietropaoli and Steve N. Georas
}

Division of Pulmonary and Critical Care Medicine, University of Rochester Medical Center, Rochester, New York, USA.

\begin{abstract}
Inflammation-associated lung injury is a major cause of morbidity and mortality for patients in intensive care units. Although the cellular and molecular events that initiate lung inflammation are now well understood, the mechanisms that promote its resolution remain poorly defined. In this issue of the JCI, D'Alessio et al. show in a mouse model that recovery from acute lung injury is not simply a passive process, but involves Tregs in an active resolution program (see the related article beginning on page 2898).
\end{abstract}

\section{Overview of lung injury}

Acute lung injury (ALI) is a syndrome defined by bilateral pulmonary infiltrates on chest radiography and arterial hypoxemia in the absence of left atrial hypertension. This syndrome has a rapidly progressive clinical presentation characterized by acute onset of dyspnea and respiratory fail-

Conflict of interest: The authors have declared that no conflict of interest exists.

Citation for this article: J. Clin. Invest. 119:2891-2894 (2009). doi:10.1172/JCI40880. ure requiring mechanical ventilation; when arterial hypoxemia is particularly severe, it is classified as acute respiratory distress syndrome (ARDS; ref. 1). ALI can be caused by direct and indirect pulmonary insults. The initial pathophysiologic events are endothelial and epithelial injury, excessive permeability of the alveolar-capillary unit, and alveolar and interstitial accumulation of proteinaceous fluid and inflammatory cells (1). This inflammatory response disrupts alveolar function, impairing fluid clearance, surfactant production, and gas exchange (2). Neutrophils are a key component of lung inflammation in ALI and are recruited to the lung by chemotactic signals produced by alveolar macrophages and other lung cell types $(3,4)$.

ALI occurs in response to a variety of acute illnesses, with common direct causes including pneumonia and aspiration of gastric contents, and common indirect causes including extrapulmonary sepsis syndrome and trauma $(5,6)$. The public health burden is high, with an incidence of approximately 80 cases per 100,000 person-years and mortality approaching $40 \%$ (7). Interestingly, persistent pulmonary inflammation and dysfunction are strongly associated with mortality (8), yet the cause of death in patients with ALI is usually multisystem organ failure instead of intractable respiratory insufficiency (9). This apparent paradox is explained by 